\title{
The surface precision of antenna impacts on its function parameter
}

\author{
Fan liang $^{1, a}$, Gao jingbo ${ }^{2, b}$ Wang cong ${ }^{2, c}$ \\ ${ }^{1}$ School of Harbin Institute of Technology, Harbin 150001, China; \\ ${ }^{2}$ School of Harbin Institute of Technology, Harbin 150001, China. \\ a894952023@qq.com bgaojb@hit.edu.cn calanwang@hit.edu.cn
}

Keywords: Antenna, Surface precision, Function parameter, Gain

\begin{abstract}
Antenna plays an important part in the satellite, the surface precision of which has an impact on its function parameter named gain. This article finds the method of decreasing the surface precision of the antenna by dividing the mesh into diverse formals. And the technique is able to improve the property of antenna, thereby, it can also contribute to the whole structure performance of satellite.
\end{abstract}

\section{Introduction}

Antenna works as an essential tool for transferring signal, which is a necessary component in the satellite. At the same time, the precision of the antenna has an influence on the property of antenna. The parameter of antenna property is influenced by the following aspects[1]: one is the structural stiffness of the antenna, the other is the surface precision, which contains four aspects: reflection principle error, reflector design error, the processing and assembling error, thermal deformation error. As for the structure of the antenna, actually, the main error is derived from two factors that includes the reflection principle error, reflector design error. The definition of reflection principle error is that there are errors between reflector composed by meshes and ideal reflector, and it is decided by the formal of the mesh and the size of the mesh. The reflector design error is that there may be a unbalanced initial stress which makes the cable net not come to the ideal situation. Due to many articles in the aspect, therefore, the article will do some researches with the reflection principle error and try to figure out the method of improving the property of the antenna.

\section{Basic principle}

As for the antenna, the two essential properties are the gain and directionality. The directionality is the ratio of maximum radiation power density and its average value on one sphere of the far-field. And the relation between the gain and the directionality is that:

$$
\begin{aligned}
& g=k d \quad \text { the efficient factor } \mathrm{k}(0 \leqslant \mathrm{k} \leqslant 1) \\
& \frac{g}{g_{0}}=\exp \left\{-\left[\frac{4 \pi \delta_{r m s}}{\lambda}\right]^{2}\right\}
\end{aligned}
$$

The $g$ represents the gain of the actual reflector, $g_{0}$ represents the gain of the ideal reflector, the $\lambda$ is the length of wave when the antenna is working, the $\delta_{r m s}$ represents the surface error of the reflector. This shows that $\delta_{r m s}$ is inversely proportional to the gain, and the minimum of the $\delta_{r m s}$ is the maximum of the gain proportion. Therefore, it can be done to control the value of the $\delta_{r m s}$ to make sure that the $\delta_{r m s}$ tends to the minimum, then the actual gain will come to the maximum. The property of the antenna will be improved mostly in this method, and the main idea of the article is based on this method.

Considering the more thin reflector, the facets will tend to the equilateral triangle, in this way, and the principle curvatures are nearly equal for any surface of revolution. Therefore, the $\delta_{r m s}$ calculation for an equilateral triangle on a spherical surface should be a good approximation for 
the actual geometry. In the case, the calculation of the $\delta_{r m s}$ is greatly simplified. At the same time, it should be done to divide the mesh of the surface into different patterns and the method can be interpreted by contrast.

By the calculation[3], it is known that the relation between the parameter of the mesh and the $\delta_{r m s}$ is given by:

$$
\frac{l}{d}=c \sqrt{\frac{\delta_{r m s}}{d} \bullet \frac{f}{d}}
$$

$\mathrm{f}$ - the focal distance of the reflector, which is half of the centre of curvature

$\mathrm{d}$-the diameter of the minimum mesh element by divided

$l$ - the side length of the polygon

c-constant

If the formula is applied for different types of meshes, the result can be figured out as follows:

Table 1 Three Facet Type comparing

\begin{tabular}{ccc}
$\begin{array}{c}\text { Facet } \\
\text { Type }\end{array}$ & c & Formula \\
\hline Hexagon & 4.046 & $\frac{l}{d}=c \sqrt{\frac{\delta_{r m s}}{d} \bullet \frac{f}{d}}$ \\
Square & 6.160 & \\
Triangle & 7.872 & \\
\hline
\end{tabular}

If the more thin reflector under consideration satisfies the condition of $\frac{f}{d}>0.5$, the divided sub- elements are equilateral polygons. There are three conclusions[3]:

One side length of the divided surface is divided equally, the number of sub-element is :

$$
m=\left(\frac{d}{l}+1\right) / 3
$$

The total number of numbers in the reflector is given by $: n_{m}=9 m^{2}-3 m$

The total number of polygon facets is given by $: n_{f}=3 m^{2}-3 m+1$

\section{Antenna structure simulation}

By applying the software named patran, according to the analysis of preceding parts of the article, dividing the parabolic antenna into diverse meshes, for example, hexagon, square ,triangle. And the result is given by the following chart:

Table 2 Comparing number of three facet types meshes

\begin{tabular}{ccccc}
\hline Facet Type & $\mathrm{m}$ & $\mathrm{n}_{\mathrm{m}}$ & $\mathrm{n}_{\mathrm{f}}$ & $\delta_{r m s}$ \\
\hline Hexagon & 20 & 3540 & 1141 & $10.983 \%$ \\
\hline Square & 23 & 4692 & 1519 & $3.568 \%$ \\
\hline Triangle & 26 & 6006 & 1951 & $1.704 \%$
\end{tabular}$\quad$\begin{tabular}{l}
$d$ \\
\hline
\end{tabular}




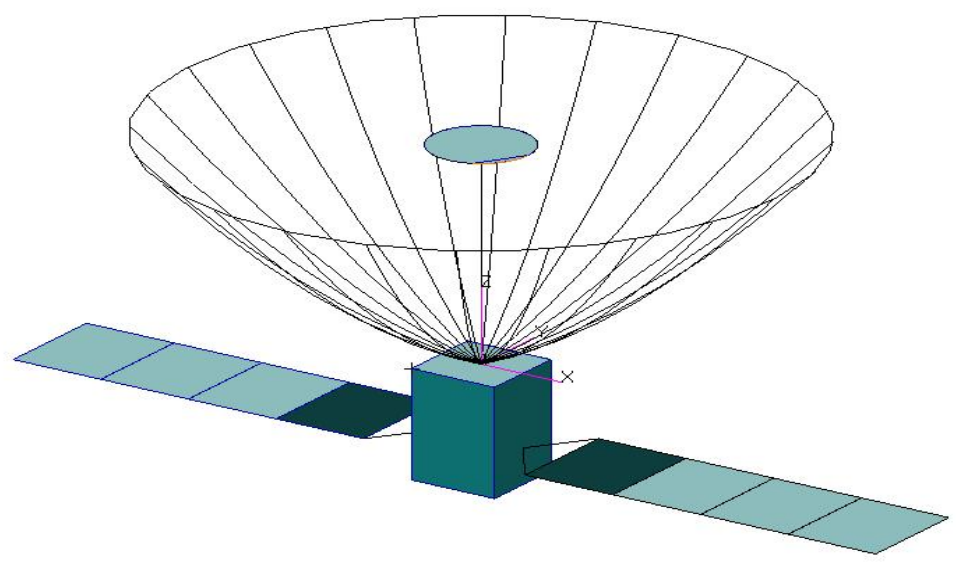

Fig.1 the overall structure of satellite

\section{Property of the antenna}

The curvature of the surface can be confirmed when the structure of the antenna is designed. Therefore, the value of the $\mathrm{f} / \mathrm{d}$ is approximate to 1 .

Through dividing the antenna into different kinds of meshes, it can be found that the size of the mesh and the number of the mesh can be confirmed when the length of every antenna has been determined. As the above figures has shown, both the total number of meshes and the density of the mesh increase apparently with decreasing the side length of sub-element. By calculating the $\delta_{r m s}$ with the above formula, it decreases greatly. As the formula appears, $\delta_{r m s}$ is inversely proportional to the gain, so, the gain of the antenna has improved greatly. There is no doubt that the property of antenna will be enhanced.

\section{Conclusion}

This article, by simulating analysis for the parabolic antenna, finds it that diverse types of meshes will have an impact on the surface error. But the actual surface error will have effects on the parameter of gain. Therefore, according to the actual situation, it should be divided the facets into different figures such as the element of the triangle, so that it will contribute to the improvement of the critical parameter of antenna. There is no doubt that the property of the antenna will be improved with the method.

\section{References}

[1] Yang dongwu, Structure Design and Profile Adjustment of Large Deployable Mesh Antenna for Satellite[D], Xian ,XiDian University, 2010.

[2] John D. Kraus \& Ronald J. Marhefka, Antennas: For All Applications Third Edition[M], Beijing: Publishing House of Electronics Industry.

[3] Agrawal P K, Anderson M S and Card M F. Preliminary design of large reflectors with flat facets [J]. IEEE Transactions on Antennas and Propagation, 1981, AP-29(4): 688一b94. 